\title{
Epidemiological and clinical characteristics of Streptococcus tigurinus endocarditis
}

\author{
María Ercibengoa', Miguel Angel Goenaga ${ }^{2}$, Carmen Ardanuy ${ }^{1,3}$, Immaculada Grau ${ }^{1,4}$, Cristina García-de-la-Maria ${ }^{5}$, \\ Manuel Almela ${ }^{6}$, Jose María Miro ${ }^{5}$, Enrique Navas ${ }^{7}$, María Carmen Fariñas ${ }^{8}$, Carlos Ruiz de Alegría ${ }^{9}$, \\ Javier de la Torre ${ }^{10}$, Fernando Fernández ${ }^{11}$, Mercedes Marín ${ }^{1,12}$, Patricia Muñoz ${ }^{1,12}$, Beatriz Orden ${ }^{13}$, \\ José Antonio Oteo ${ }^{14}$, Lara García-Álvarez ${ }^{14}$, Arístides de Alarcón ${ }^{15}$, José Antonio Lepe Jiménez ${ }^{15}$ \\ and Jose María Marimón ${ }^{1,16^{*}}$
}

\begin{abstract}
Background: Streptococcus tigurinus was recently described as a new streptococcal species within the viridans group streptococci (VGS). The objectives of the present work were to analyse the clinical and microbiological characteristics of $\mathrm{S}$. tigurinus isolated from patients with bacteraemias, to determine the prevalence of $\mathrm{S}$. tigurinus among VGS endocarditis in Spain, and to compare the clinical characteristics and outcomes of endocarditis caused by S. tigurinus and other VGS.

Methods: Retrospective nationwide study, performed between 2008 and 2016 in 9 Spanish hospitals from 7 different provinces comprising 237 cases of infective endocarditis. Streptococcal isolates were identified by sequencing fragments of their $16 \mathrm{~S}$ rRNA, sodA and groEL genes. Clinical data of patients with streptococcal endocarditis were prospectively collected according to a pre-established protocol.

Results: Patients with endocarditis represented $7 / 9$ (77.8\%) and 26/86 (30.2\%) of the bacteraemias caused by S. tigurinus and other VGS, respectively $(p<0.001)$, in two of the hospital participants. Among patients with streptococcal endocarditis, 12 different Streptococcus species were recognized being S. oralis, S. tigurinus and S. mitis the three more common. No relevant statistical differences were observed in the clinical characteristics and outcomes of endocarditis caused by the different VGS species.

Conclusions: In this multicenter study performed in Spain, S. tigurinus showed a higher predilection for the endocardial endothelium as compared to other VGS. However, clinical characteristics and outcomes of endocarditis caused by S. tigurinus did not significantly differ from endocarditis caused by other oral streptococci.
\end{abstract}

Keywords: Streptococcus Viridans, Infective endocarditis, Multicentre studies, Epidemiologic surveillance, Antibiotic resistance

\section{Background}

Streptococcus tigurinus was firstly described as a new streptococcal species in 2012 based on DNA hybridization analysis and $16 \mathrm{~S}$ rRNA sequencing, being genetically very similar to Streptococcus oralis [1]. In fact, in 2016, and based on whole-genome sequencing, it was proposed to classify S. tigurinus as a Streptococcus oralis subspecies [2].

\footnotetext{
* Correspondence: josemaria.marimonortizdez@osakidetza.eus

${ }^{1}$ CIBER Enfermedades Respiratorias-CIBERES, Madrid, Spain

${ }^{16}$ Servicio de Microbiología, Hospital Universitario Donostia-IIS Biodonostia,

Paseo Dr Beguiristain s/n, 20014 San Sebastián, Spain

Full list of author information is available at the end of the article
}

S. tigurinus, as other mitis group streptococci has been found as a commensal of the human oral cavity although since its initial description it has been also documented as a causative agent of infective endocarditis (IE) and other infections as meningitis, spondylodiscitis, osteomyelitis, prosthetic infections, etc. [1, 3-7]. The objective of the present study was to establish the clinical and microbiological characteristics of S. tigurinus isolated from patients with bacteraemias, to determine the prevalence of S. tigurinus among the cases of viridans group streptococci (VGS) endocarditis in Spain, and to

(c) The Author(s). 2019 Open Access This article is distributed under the terms of the Creative Commons Attribution 4.0 International License (http://creativecommons.org/licenses/by/4.0/), which permits unrestricted use, distribution, and reproduction in any medium, provided you give appropriate credit to the original author(s) and the source, provide a link to the Creative Commons license, and indicate if changes were made. The Creative Commons Public Domain Dedication waiver (http://creativecommons.org/publicdomain/zero/1.0/) applies to the data made available in this article, unless otherwise stated. 
compare the outcomes of IE caused by $S$. tigurinus and by other VGS.

\section{Methods}

\section{S. tigurinus prevalence study}

To study the relevance of finding S. tigurinus in a blood culture, all VGS isolated from blood cultures collected between 2008 and 2016 in two hospitals from Barcelona and San Sebastian, two cities from the north of Spain separated more than $500 \mathrm{~km}$ apart, were classified by phenotypic methods and all $S$. oralis were further identified by gene sequencing.

\section{S. tigurinus endocarditis study}

To analyse the characteristics of IE caused by $S$. tigurinus, a retrospective nationwide study, performed between 2008 and 2016 in 9 Spanish hospitals located in 7 different provinces comprising 237 cases of IE was performed. Diagnosis of IE was done according to the revised Duke diagnostic criteria [8]. Clinical IE data were prospectively collected according to a pre-established protocol [9]. Only cases with definite IE diagnosis were included in the study.

\section{Microbiological techniques}

VGS isolates were identified by sequencing fragments of their $16 \mathrm{~S}$ rRNA, sodA and groEL genes and comparing them with those available at the NCBI and LeBibi databases [10]. A similitude of $>99 \%$ with the 3 genes was considered for a correct species identification. All S. tigurinus detected were genotyped by MLST according to the established protocol at the Oral Streptococcus MLST Database web page (https://pubmlst.org/ oralstrep). Antimicrobial MICs were determined by the broth microdilution method using Iso-Sensitest Broth (Oxoid) supplemented with lysed horse blood 5\% $v / \mathrm{v}$. After incubation for $24 \mathrm{~h}$ at $35^{\circ} \mathrm{C}$, susceptibility results were read and interpreted according to CLSI guidelines. S. pneumoniae ATCC 49619 was used as control.

\section{Statistical analysis}

The unpaired t-test or the chi-square test (Fisher's exact test when appropriate) was used to compare continuous and categorical variables, respectively. All statistical analyses were performed using the online available GraphPad software (www.graphpad.com/quickcalcs/).

\section{Ethical considerations}

The project and the common case report form were approved by the national and local institutional review boards and ethics committees (E.C. 18/07) and all patients gave their written informed consent to participate in the study.

\section{Results}

In the present work, 169 VGS isolates were identified by molecular methods: 95 from the prevalence study and 74 from the study of IE. The $16 \mathrm{~S}$ rRNA gene correctly identified all of them, sodA misidentified one $S$. oralis isolate as $S$. tigurinus and groEL misidentified $5 S$. oralis isolates as S. tigurinus $(n=2)$, S. cristatus $(\mathrm{n}=2)$ and S. mitis $(n=1)$.

\section{S. tigurinus prevalence study}

Overall, 95 cases of VGS bacteraemias recorded in the hospitals of Barcelona and San Sebastian were studied, being 9 identified as $S$. tigurinus. Patients with endocarditis represented $7 / 9$ (77.8\%) of the S. tigurinus and $26 / 86(30.2 \%)$ of the remaining VGS $(p=0.008)$. This data suggests a bigger attraction of $S$. tigurinus for the endocardial endothelium as compared with related species of the VGS, a finding that has been also observed in other studies [6].

\section{S. tigurinus endocarditis study}

Of the 237 IE caused by VGS recorded in the study, 74 isolates were available for further studies and were identified by phenotypic methods and by gene sequencing. In global, 12 different Streptococcus species were recognized being $S$. oralis, S. tigurinus and S. mitis the three more common causing $37.8,23.0$ and $21.6 \%$ of IE cases respectively (Table 1). All S. tigurinus had been previously identified by phenotypic methods as $S$. oralis and were found in $5 / 9$ hospitals and in 4/7 provinces. All $S$. tigurinus were fully susceptible to oral penicillin, amoxicillin, and cefotaxime except one isolate that had a penicillin $\mathrm{MIC}=0.12 \mathrm{mg} /$ $\mathrm{L}$. Three isolates were tetracycline-resistant $(\mathrm{MIC}>4 \mathrm{mg} / \mathrm{L}$ ) and another three erythromycin-resistant $(\mathrm{MIC}=2 \mathrm{mg} / \mathrm{L}$ ). All S. tigurinus isolates were susceptible to clindamycin, levofloxacin and vancomycin. A large heterogeneity of $S$. tigurinus was observed by MLST, having all different ST. Among the 17 S. tigurinus isolates, there were only two ST

Table 1 Species of viridans group streptococci causing infective endocarditis in Spain, 2008-2016

\begin{tabular}{lll}
\hline Species & $\mathrm{n}$ & $\%$ \\
\hline S. oralis & 28 & $37.8 \%$ \\
S. tigurinus & 17 & $23.0 \%$ \\
S. mitis & 16 & $21.6 \%$ \\
S. parasanguinis & 3 & $4.1 \%$ \\
S. sanguinis & 2 & $2.7 \%$ \\
S. pneumoniae & 2 & $2.7 \%$ \\
S. infantis & 1 & $1.4 \%$ \\
S. salivarius & 1 & $1.4 \%$ \\
S. infantarius & 1 & $1.4 \%$ \\
S. gordonii & 1 & $1.4 \%$ \\
S. anginosus & 1 & $1.4 \%$ \\
S. alactolyticus & 1 & $1.4 \%$ \\
Total & 74 & \\
\hline
\end{tabular}


Table 2 Clinical data of patients with infective endocarditis caused by S. mitis, S. oralis, S. tigurinus and other viridans group streptococci, Spain, 2008-2016

\begin{tabular}{|c|c|c|c|c|}
\hline & S. mitis $(n=16)$ & S. oralis $(n=28)$ & S. tigurinus $(n=17)$ & Other VGS $(n=13)$ \\
\hline \multicolumn{5}{|l|}{ Demographics } \\
\hline Age in years: average \pm SD (range) & $58.4 \pm 17.5(35-94)$ & $60.0 \pm 16.7(32-86)$ & $66.7 \pm 17.2(19-87)$ & $61.2 \pm 13.9(28-80)$ \\
\hline Female/Male & $2 / 14$ & $4 / 24$ & $6 / 11$ & $5 / 8$ \\
\hline \multicolumn{5}{|l|}{ Underlying conditions } \\
\hline Diabetes mellitus & 2 & 2 & 0 & 0 \\
\hline Renal insufficiency & 0 & 1 & 2 & 1 \\
\hline Pulmonary disease & 3 & 2 & 3 & 1 \\
\hline Neoplasm & 2 & 2 & 3 & 1 \\
\hline HIV infection & 0 & 3 & 0 & 1 \\
\hline \multicolumn{5}{|l|}{ Risk factors } \\
\hline Previous infective endocarditis & 2 & 5 & 2 & 1 \\
\hline Heart failure & 1 & 4 & 4 & 2 \\
\hline Atrial fibrillation & 3 & 4 & 2 & 1 \\
\hline \multicolumn{5}{|l|}{ Site of acquisition } \\
\hline Nosocomial & 0 & 0 & 0 & 0 \\
\hline Community -acquired & 15 & 27 & 16 & 12 \\
\hline Health care-related & 1 & 1 & 1 & 1 \\
\hline \multicolumn{5}{|l|}{ Symptoms at admission } \\
\hline \multicolumn{5}{|l|}{ Affected valve } \\
\hline Aortic & 6 & 14 & 7 & 7 \\
\hline Mitral & 6 & 9 & 6 & 2 \\
\hline Aortic + mitral & 2 & 3 & 2 & 2 \\
\hline Tricuspid & 1 & 0 & 0 & 0 \\
\hline Tricuspid + mitral & 0 & 0 & 0 & 1 \\
\hline Aortic + tricuspid + mitral & 0 & 1 & 0 & 0 \\
\hline Pulmonary & 1 & 0 & 0 & 0 \\
\hline Ductus arteriosus & 0 & 0 & 0 & 1 \\
\hline Not determined & 0 & 1 & 2 & 0 \\
\hline \multicolumn{5}{|l|}{ Presentation } \\
\hline Fever $>38^{\circ} \mathrm{C}$ & 16 & 23 & $13 / 16^{\mathrm{a}}$ & 11 \\
\hline Splinter hemorrhages & 1 & 0 & 0 & 2 \\
\hline Osler nodes & 0 & 0 & 0 & 1 \\
\hline Janeway lesions & 1 & 0 & 0 & 1 \\
\hline New murmur & 3 & 15 & $5 / 16$ & 8 \\
\hline Worsening of old murmur & 0 & $4 / 24$ & $1 / 15$ & 0 \\
\hline Protein $C$ reactive: average \pm SD (range) & $74.2 \pm 41.5(13-168)$ & $55.7 \pm 45.6(1-138)$ & $52.3 \pm 96.4(3-356)$ & $40.8 \pm 42.7(3-101)$ \\
\hline Elevated Rheumatoid factor & $0 / 2$ & $4 / 11$ & $1 / 4$ & $2 / 4$ \\
\hline \multicolumn{5}{|l|}{ Vegetations } \\
\hline Not found & 6 & 6 & 6 & 4 \\
\hline Aortic & 4 & 10 & 6 & 5 \\
\hline Mitral & 3 & 7 & 3 & 1 \\
\hline Tricuspid & 1 & 0 & 0 & 0 \\
\hline Aortic + mitral & 1 & 5 & 2 & 2 \\
\hline
\end{tabular}


Table 2 Clinical data of patients with infective endocarditis caused by S. mitis, S. oralis, S. tigurinus and other viridans group streptococci, Spain, 2008-2016 (Continued)

\begin{tabular}{|c|c|c|c|c|}
\hline & S. mitis $(n=16)$ & S. oralis $(n=28)$ & S. tigurinus $(n=17)$ & Other VGS $(n=13)$ \\
\hline Mitral + tricuspid & 0 & 0 & 0 & 1 \\
\hline Chordae tendinae & 1 & 0 & 0 & 0 \\
\hline \multicolumn{5}{|l|}{ Intracardiac complications } \\
\hline Perforation & 2 & 4 & 3 & 3 \\
\hline Abscess & 1 & 4 & 1 & 2 \\
\hline Pseudoaneurysm & 0 & 1 & 0 & 1 \\
\hline Pseudoaneurysm \& perforation & 0 & 2 & 0 & 0 \\
\hline NC & 0 & 1 & 0 & 2 \\
\hline \multicolumn{5}{|l|}{ Clinical course } \\
\hline Embolism & 1 & $6 / 27^{a}$ & $5 / 16^{\mathrm{a}}$ & 4 \\
\hline New heart failure & 1 & $12 / 27^{\mathrm{a}}$ & 5 & 4 \\
\hline Persistent bacteraemia & 1 & 0 & 0 & 1 \\
\hline \multicolumn{5}{|l|}{ Surgery } \\
\hline Indicated & 5 & $19 / 27^{a}$ & 9 & 7 \\
\hline Performed & 5 & $18 / 27^{\mathrm{a}}$ & 6 & 6 \\
\hline \multicolumn{5}{|l|}{ Criteria for surgery $^{\mathrm{b}}$} \\
\hline Cardiac insufficiency & 2 & 9 & 2 & 3 \\
\hline Early prosthetic IE & 0 & 1 & 0 & 0 \\
\hline Late prosthetic IE & 1 & 2 & 0 & 0 \\
\hline Valve insufficiency & 3 & 10 & 3 & 4 \\
\hline Embolisms & 0 & 1 & 0 & 1 \\
\hline Others & 1 & 5 & 3 & 1 \\
\hline \multicolumn{5}{|l|}{ Outcome } \\
\hline Days hospital stay: average \pm SD (range) & $32.4 \pm 19.3(9-85)$ & $31.0 \pm 21.8(3-106)$ & $29.3 \pm 17.9(5-74)$ & $29.9 \pm 16.3(6-53)$ \\
\hline In- hospital mortality & 0 & 4 & 2 & 2 \\
\hline 1-year mortality ${ }^{c}$ & $0 / 14$ & $3 / 22$ & $2 / 15$ & $1 / 8$ \\
\hline Recurrence & 0 & 2 & 2 & 0 \\
\hline \multicolumn{5}{|l|}{ Antibiotic treatment } \\
\hline Beta-lactams alone & 6 & 9 & 6 & 5 \\
\hline Beta-lactams + gentamicin & 8 & 12 & 7 & 6 \\
\hline Other combinations & 2 & 7 & 4 & 2 \\
\hline
\end{tabular}

${ }^{\mathrm{a} D e n o m i n a t o r}$ adjusted to patients with data available

bome patients had more than one criteria for surgery

${ }^{\mathrm{C}}$ Excluding patents died at hospital

previously defined in the MLST database, ST30 and ST65, both previously identified as $S$. oralis from patients with gingivitis. Comparing IE caused by $S$. tigurinus and other VGS, patients' average age was higher for S. tigurinus endocarditis, without statistical significance ( $p=0.179$ compared to $S$. mitis) (Table 2). No relevant statistical differences were observed in the clinical characteristics of IE caused by the different VGS species. Left heart valves were more frequently affected in S. tigurinus IE as well as in other streptococci: $45.9 \%$ mitral, $31.1 \%$ aortic, and $13.6 \%$ both valves. Considering all VGS IE, perforation was the most common intracardiac complication (16.2\%), followed by abscess $(10.8 \%)$ and pseudoaneurysm alone $(2.7 \%)$ or with perforation (2.7\%). In-hospital overall mortality in VGS IE (10.8\%) was relatively high as compared with other studies [11]. Mortality due to S. tigurinus was quite similar to that of $S$. oralis $(11.3 \%$ vs $14.8 \%, p=1)$. Surprisingly, no mortality was recorded for any of the 16 patients with $S$. mitis IE that was also the group with less patients requiring surgical treatment. Of the patients with IE caused by VGS, 22 were treated only with betalactams (20 with ceftriaxone, 1 with ceftriaxone and ampicillin and 1 
with imipenem), 26 with betalactams and gentamicin, being ceftriaxone-gentamicin the combination most frequently used (in 10 patients ceftriaxone-gentamicin alone and in another 8 with a third antibiotic). There were no differences in the antibiotic treatment despite the species identified.

\section{Discussion}

Despite the advances in imaging (echocardiography and nuclear medicine), molecular microbiology and surgery, IE is still today a serious disease with high morbidity and mortality rates. In high-income countries, epidemiology of IE is changing with an increase of elderly patients with prosthetic valves or implantable cardiovascular devices [11]. Also, etiologic agents causing IE seems to be changing, with an increase of staphylococcal IE and a reduction of IE caused by VGS $[11,12]$. In this Spanish multicenter study, it was previously shown that VGS represented $27.5 \%$ cases of definitive IE [13]. In the present work it has been shown that $S$. tigurinus was responsible for $20 \%$ of these definitive IE cases caused by VGS.

S. tigurinus has been associated to IE since its first description, although it has been also described as causing meningitis, spondylodiscitis, prosthetic infections, osteomyelitis, and periodontitis among others. However, in a recent review, IE was the most commonly reported manifestation of S. tigurinus infection [6] probably because IE has been more systematically searched for than other kind of infections. In that review, no deaths were documented among patients with $S$. tigurinus infection except for one case of osteomyelitis. In our study, 2 patients with $S$. tigurinus IE died during admission with no difference in mortality rates to IE caused by other VGS.

Besides to the oral origin of S. tigurinus infections, an enteric source has been also postulated after the translocation of the pathogen from an intraabdominal disorder [14]. However we considered that in most of our patients the origin was the oral cavity as no intraabdominal condition was found in any of the patients, whether the causative agent of the IE was $S$. tigurinus or another of the VSG commonly found in the oral mucosa. The oral origin of most VGS IE highlights the need of an exquisite dental care in patients with risk for IE [15].

IE caused by $S$. tigurinus did not clinically differ from IE caused by other VGS, showing a community-acquired origin, clinical course and outcomes in general better than bacterial IE caused by other Gram-positive bacteria as Staphylococcus aureus or Enterococcus [11]. Despite the wide genomic heterogeneity most isolates were fully susceptible to commonly used antibiotics in the treatment of IE. An endocarditis should always be suspected when a $S$. tigurinus is isolated from a blood culture due to the high prevalence of IE caused by this, otherwise commensal bacteria.

\section{Conclusion}

In this multicenter study performed in Spain, S. tigurinus was a common cause of IE. Clinical characteristics and outcomes of S. tigurinus endocarditis did not differ from endocarditis caused by other VGS. S. tigurinus showed a high genomic heterogeneity with most isolates susceptible in vitro to antibiotics commonly used in the treatment of IE.

\section{Abbreviations}

CLSI: Clinical and laboratory standards Institute; IE: Infective endocarditis; MIC: Minimum inhibitory concentration; MLST: Multi-locus sequence typing; ST: Sequence-type; VGS: Viridans group streptococci

\section{Acknowledgements}

Members of the GAMES Group (Spanish Collaboration on Endocarditis Group): Hospital Costa del Sol, (Marbella): Fernando Fernández Sánchez, Mariam Noureddine, Gabriel Rosas, Javier de la Torre Lima; Hospital Universitario de Cruces, (Bilbao): José Aramendi, María José Blanco, Roberto Blanco, María Victoria Boado, Marta Campaña Lázaro, Alejandro Crespo, Josune Goikoetxea, José Ramón Iruretagoyena, Josu Irurzun Zuazabal, Leire López-Soria, Miguel Montejo, Javier Nieto, David Rodrigo, David Rodríguez, Regino Rodríguez, Yolanda Vitoria, Roberto Voces; Hospital Universitario Virgen de la Victoria, (Málaga): Ma Victoria García López, Radka Ivanova Georgieva, Guillermo Ojeda, Isabel Rodríguez Bailón, Josefa Ruiz Morales; Hospital Universitario DonostiaPoliclínica Gipuzkoa, (San Sebastián): Ana María Cuende, Tomás Echeverría, Ana Fuerte, Eduardo Gaminde, Miguel Ángel Goenaga, Pedro Idígoras, José Antonio Iribarren, Alberto Izaguirre Yarza, Xabier Kortajarena Urkola, Carlos Reviejo; Hospital General Universitario de Alicante, (Alicante): Rafael Carrasco, Vicente Climent, Patricio Llamas, Esperanza Merino, Joaquín Plazas, Sergio Reus; Complejo Hospitalario Universitario A Coruña, (A Coruña): Nemesio Álvarez, José María Bravo-Ferrer, Laura Castelo, José Cuenca, Pedro Llinares, Enrique Miguez Rey, María Rodríguez Mayo, Efrén Sánchez, Dolores Sousa Regueiro; Complejo Hospitalario Universitario de Huelva, (Huelva): Francisco Javier Martínez; Hospital Universitario de Canarias, (Canarias): Ma del Mar Alonso, Beatriz Castro, Dácil García Rosado, Ma del Carmen Durán, Ma Antonia Miguel Gómez, Juan Lacalzada, Ibrahim Nassar; Hospital Regional Universitario de Málaga, (Málaga): Antonio Plata Ciezar, José Ma Reguera Iglesias; Hospital Universitario Central Asturias, (Oviedo): Víctor Asensi Álvarez, Carlos Costas, Jesús de la Hera, Jonnathan Fernández Suárez, Lisardo Iglesias Fraile, Víctor León Arguero, José López Menéndez, Pilar Mencia Bajo, Carlos Morales, Alfonso Moreno Torrico, Carmen Palomo, Begoña Paya Martínez, Ángeles Rodríguez Esteban, Raquel Rodríguez García, Mauricio Telenti Asensio; Hospital Clínic-IDIBAPS, Universidad de Barcelona, (Barcelona): Manuel Almela, Juan Ambrosioni, Manuel Azqueta, Mercè Brunet, Marta Bodro, Ramón Cartañá, Carlos Falces, Guillermina Fita, David Fuster, Cristina García de la Mària, Marta Hernández-Meneses, Jaume Llopis Pérez, Francesc Marco, José M. Miró, Asunción Moreno, David Nicolás, Salvador Ninot, Eduardo Quintana, Carlos Paré, Daniel Pereda, Juan M. Pericás, José L. Pomar, José Ramírez, Irene Rovira, Elena Sandoval, Marta Sitges, Dolors Soy, Adrián Téllez, José M. Tolosana, Bárbara Vidal, Jordi Vila; Hospital General Universitario Gregorio Marañón, (Madrid): Iván Adán, Javier Bermejo, Emilio Bouza, Daniel Celemín, Gregorio Cuerpo Caballero, Antonia Delgado Montero, Ana Fernández Cruz, Ana García Mansilla, Ma Eugenia García Leoni, Víctor González Ramallo, Martha Kestler Hernández, Amaia Mari Hualde, Mercedes Marín, Manuel Martínez-Sellés, Ma Cruz Menárguez, Patricia Muñoz, Cristina Rincón, Hugo Rodríguez-Abella, Marta Rodríguez-Créixems, Blanca Pinilla, Ángel Pinto, Maricela Valerio, Pilar Vázquez, Eduardo Verde Moreno; Hospital Universitario La Paz, (Madrid): Isabel Antorrena, Belén Loeches, Alejandro Martín Quirós, Mar Moreno, Ulises Ramírez, Verónica Rial Bastón, María Romero, Araceli Saldaña; Hospital Universitario Marqués de Valdecilla, (Santander): Jesús Agüero Balbín, Carlos Armiñanzas Castillo, Ana Arnaiz, Francisco Arnaiz de las Revillas, Manuel Cobo Belaustegui, María Carmen Fariñas, Concepción Fariñas-Álvarez, Rubén Gómez Izquierdo, Iván García, Claudia González Rico, Manuel Gutiérrez-Cuadra, José Gutiérrez Díez, Marcos Pajarón, José Antonio Parra, Ramón Teira, Jesús Zarauza; Hospital Universitario Puerta de Hierro, (Madrid): Fernando Domínguez, Pablo García Pavía, Jesús González, Beatriz Orden, Antonio Ramos; Hospital Universitario Ramón y Cajal, (Madrid): Tomasa Centella, José Manuel Hermida, José Luis Moya, Pilar Martín-Dávila, Enrique Navas, Enrique Oliva, Alejandro del Río, Soledad Ruiz; Hospital Universitario Virgen de las Nieves, (Granada): Carmen 
Hidalgo Tenorio; Hospital Universitario Virgen Macarena, (Sevilla): Manuel Almendro Delia, Omar Araji, José Miguel Barquero, Román Calvo Jambrina, Marina de Cueto, Juan Gálvez Acebal, Irene Méndez, Isabel Morales, Luis Eduardo López-Cortés; Hospital Universitario Virgen del Rocío, (Sevilla): Arístides de Alarcón, Emilio García, Juan Luis Haro, José Antonio Lepe, Francisco López, Rafael Luque; Hospital San Pedro, (Logroño): Luis Javier Alonso, Pedro Azcárate, José Manuel Azcona Gutiérrez, José Ramón Blanco, Antonio Cabrera Villegas, Lara García-Álvarez, José Antonio Oteo, Mercedes Sanz; Hospital de la Santa Creu i Sant Pau, (Barcelona): Natividad de Benito, Mercé Gurguí, Cristina Pacho, Roser Pericas, Guillem Pons; Complejo Hospitalario Universitario de Santiago de Compostela, (A Coruña): M. Álvarez, A. L. Fernández, Amparo Martínez, A. Prieto, Benito Regueiro, E. Tijeira, Marino Vega; Hospital Santiago Apóstol, (Vitoria): Andrés Canut Blasco, José Cordo Mollar, Juan Carlos Gainzarain Arana, Oscar García Uriarte, Alejandro Martín López, Zuriñe Ortiz de Zárate, José Antonio Urturi Matos; Hospital SAS Línea de la Concepción, (Cádiz): Gloria García Domínguez, Antonio Sánchez-Porto; Hospital Clínico Universitario Virgen de la Arrixaca (Murcia): José Ma Arribas Leal, Elisa García Vázquez, Alicia Hernández Torres, Ana Blázquez, Gonzalo de la Morena Valenzuela; Hospital de Txagorritxu, (Vitoria): Ángel Alonso, Javier Aramburu, Felicitas Elena Calvo, Anai Moreno Rodríguez, Paola Tarabini-Castellani; Hospital Virgen de la Salud, (Toledo): Eva Heredero Gálvez, Carolina Maicas Bellido, José Largo Pau, Ma Antonia Sepúlveda, Pilar Toledano Sierra, Sadaf Zafar labal-Mirza; Hospital Rafael Méndez, (Lorca-Murcia):, Eva Cascales Alcolea, Ivan Keituqwa Yañez, Ana Peláez Ballesta; Hospital Universitario San Cecilio (Granada): Eduardo Moreno Escobar, Alejandro Peña Monje, Valme Sánchez Cabrera, David Vinuesa García; Hospital Son Llátzer (Palma de Mallorca): María Arrizabalaga Asenjo, Carmen Cifuentes Luna, Juana Núñez Morcillo, Ma Cruz Pérez Seco, Aroa Villoslada Gelabert; Hospital Universitario Miguel Servet (Zaragoza): Carmen Aured Guallar, Nuria Fernández Abad, Pilar García Mangas, Marta Matamala Adell, Ma Pilar Palacián Ruiz, Juan Carlos Porres; Hospital General Universitario Santa Lucía (Cartagena): Begoña Alcaraz Vidal, Nazaret Cobos Trigueros, María Jesús Del Amor Espín, José Antonio Giner Caro, Roberto Jiménez Sánchez, Amaya Jimeno Almazán, Alejandro Ortín Freire, Monserrat Viqueira González; Hospital Universitario Son Espases (Palma de Mallorca): Pere Pericás Ramis, Ma Ángels Ribas Blanco, Enrique Ruiz de Gopegui Bordes, Laura Vidal Bonet; Complejo Hospitalario Universitario de Albacete (Albacete): Ma Carmen Bellón Munera, Elena Escribano Garaizabal, Antonia Tercero Martínez, Juan Carlos Segura Luque.

\section{Funding}

JMM received a personal 80:20 research grant from the Institut d'Investigacions Biomèdiques August Pi i Sunyer (IDIBAPS), Barcelona, Spain during 2017-19. No funding entity played any role in the design of the study and data collection, analysis, and interpretation of data and in writing the manuscript.

\section{Availability of data and materials}

The datasets generated and analysed during the current study are not publicly available due to confidently but are available from the GAMES project on reasonable request.

\section{Authors' contributions}

ME, JMM performed bacterial molecular techniques (identification and genotyping) and antimicrobial susceptibility testing and contributed to the study conception, data acquisition, analysis and interpretation of findings, and drafting of the manuscript. MAG, CA, IG, CG, MA, JMM, EN, MCF, CRA, JT, FF, $\mathrm{MM}, \mathrm{PM}, \mathrm{BO}, \mathrm{JAO}, \mathrm{LGA}, \mathrm{AA}, \mathrm{JAL}$, contributed to the study by recording clinical data, collecting microbial isolates, interpreting of findings, and drafting of the manuscript. All authors have read and approved the manuscript.

\section{Ethics approval and consent to participate}

The project and the common case report form were approved by the national and local institutional review boards and ethics committees (E.C. 18/07). All patients gave their written informed consent to participate in the study.

\section{Consent for publication}

Not applicable.

\section{Competing interests}

JMM has received consulting honoraria and/or research grants from AbbVie, Angelini, Bristol-Myers Squibb, Jansen, Genentech, Medtronic, Merck, Novartis, Gilead Sciences, and ViiV Healthcare outside the submitted work. All other authors: none to declare.

\section{Publisher's Note}

Springer Nature remains neutral with regard to jurisdictional claims in published maps and institutional affiliations.

\section{Author details}

${ }^{1}$ CIBER Enfermedades Respiratorias-CIBERES, Madrid, Spain. ${ }^{2}$ Servicio de Enfermedades Infecciosas, Hospital Universitario, Donostia, Spain. ${ }^{3}$ Servicio de Microbiologia, Hospital Universitari de Bellvitge. Institut d'Investigació Biomèdica de Bellvitge (IDIBELL), University of Barcelona, Barcelona, Spain. ${ }^{4}$ Servicio de Enfermedades Infecciosas, Hospital Universitari de Bellvitge. Institut d' Investigació Biomèdica de Bellvitge (IDIBELL), University of Barcelona, Barcelona, Spain. ${ }^{5}$ Servicio de Enfermedades Infecciosas, Hospital Clínic de Barcelona, Institut d' Investigacions Biomèdiques August Pi i Sunyer (IDIBAPS), University of Barcelona, Barcelona, Spain. '5ervicio de Microbiología, Hospital Clínic de Barcelona, Institut d' Investigacions Biomèdiques August Pi i Sunyer (IDIBAPS), Barcelona, Spain. ${ }^{7}$ Servicio de Enfermedades Infecciosas, Hospital Universitario Ramón y Cajal, Madrid, Spain. ${ }^{8}$ Servicio de Enfermedades Infecciosas, Hospital Universitario Marqués de Valdecilla, Universidad de Cantabria, Santander, Spain. ${ }^{9}$ Servicio de Microbiología, Hospital Universitario Marqués de Valdecilla, Universidad de Cantabria, Santander, Spain. ${ }^{10}$ Grupo de Enfermedades Infecciosas de la Unidad de Medicina Interna, Hospital Costa del Sol, Marbella, Málaga, Spain. ${ }^{11}$ Servicio de Microbiología, Hospital Costa del Sol, Marbella, Málaga, Spain. ${ }^{12}$ Departamento de Medicina, Servicio de Microbiología y Enfermedades Infecciosas, Hospital General Universitario Gregorio Marañón, Facultad de Medicina, Universidad Complutense de Madrid, Instituto de Investigación Sanitaria Gregorio, Marañón, Madrid, Spain. ${ }^{13}$ Departamento de Microbiología, Hospital Universitario Puerta de Hierro Majadahonda, Madrid, Spain. ${ }^{14}$ Departamento de Enfermedades Infecciosas, Hospital Universitario San Pedro, Logroño, La Rioja, Spain. ${ }^{15}$ Clinical Unit of Infectious Diseases, Microbiology and Preventive Medicine. Infectious Diseases Research Group, Institute of Biomedicine of Seville (IBIS), University of Seville/CSIC/University Virgen del Rocío and Virgen Macarena, Sevilla, Spain. ${ }^{16}$ Servicio de Microbiología, Hospital Universitario Donostia-IIS Biodonostia, Paseo Dr Beguiristain s/n, 20014 San Sebastián, Spain.

Received: 16 May 2018 Accepted: 18 March 2019

Published online: 29 March 2019

\section{References}

1. Zbinden A, Mueller NJ, Tarr PE, Spröer C, Keller PM, Bloemberg GV. Streptococcus tigurinus sp. nov., isolated from blood of patients with endocarditis, meningitis and spondylodiscitis. Int J Syst Evol Microbiol. 2012; 62:2941-5.

2. Jensen A, Scholz CF, Kilian M. Re-evaluation of the taxonomy of the mitis group of the genus Streptococcus based on whole genome phylogenetic analyses, and proposed reclassification of Streptococcus dentisani as Streptococcus oralis subsp. dentisani comb. nov., Streptococcus tigurinus as Streptococcus oralis subsp. tigurinus comb. nov., and Streptococcus oligofermentans as a later synonym of Streptococcus cristatus. Int J Syst Evol Microbiol. 2016;66:4803-20.

3. Zbinden A, Aras F, Zbinden R, Mouttet F, Schmidlin PR, Bloemberg GV, et al. Frequent detection of Streptococcus tigurinus in the human oral microbial flora by a specific 165 rRNA gene real-time TaqMan PCR. BMC Microbiol. 2014;14:231.

4. Conrads G, Barth S, Möckel M, Lenz L, van der Linden M, Henne K. Streptococcus tigurinus is frequent among gtfR-negative Streptococcus oralis isolates and in the human oral cavity, but highly virulent strains are uncommon. J Oral Microbiol. 2017:9:1307079.

5. Michelena A, Bonavila C, Zubeltzu B, Goenaga MA. Endocarditis due to Streptococcus tigurinus: presentation of a case and a review of the literature. Enferm Infecc Microbiol Clin. 2015;33:575-6.

6. Hirai J, Sakanashi D, Hagihara M, Haranaga S, Uechi K, Kato H, et al. Bacteremia due to Streptococcus tigurinus: a case report and literature review. J Infect Chemother. 2016;22:762-6.

7. Kim B, Huh HJ, Chung DR, Kim WS, Ki CS, Lee NY. The first case of concurrent infective endocarditis and spondylitis caused by Streptococcus tigurinus. Ann Lab Med. 2015;35:654-6.

8. Li JS, Sexton DJ, Mick N, Nettles R, Fowler VG Jr, Ryan T, et al. Proposed modifications to the Duke criteria for the diagnosis of infective endocarditis. Clin Infect Dis. 2000;30:633-8. 
9. Muñoz P, Kestler M, De Alarcon A, Miro JM, Bermejo J, Rodríguez-Abella $H$ et al. Current epidemiology and outcome of infective endocarditis: a multicenter, prospective, cohort study. Medicine (Baltimore). 2015;94:e1816

10. Glazunova O, Raoult D, Roux V. Partial sequence comparison of the rpoB, sodA, groEL and gyrB genes within the genus Streptococcus. Int I Syst Evol Microbiol. 2009:59:2317-22.

11. Hill EE, Herijgers P, Claus P, Vanderschueren S, Herregods MC, Peetermans WE. Infective endocarditis: changing epidemiology and predictors of 6-month mortality: a prospective cohort study. Eur Heart J. 2007;28:196-203.

12. Ambrosioni J, Hernandez-Meneses M, Téllez A, Pericàs J, Falces C, Tolosana $J \mathrm{M}$, et al. The changing epidemiology of infective endocarditis in the twenty-first century. Curr Infect Dis Rep. 2017;19:21.

13. Vicent L, Saldivar HG, Bouza E, Muñoz P, Cuerpo G, de Alarcón A, et al. Prognostic implications of a negative echocardiography in patients with infective endocarditis. Eur J Intern Med. 2018; Epub ahead of print.

14. Bourassa L, Clarridge JE 3rd. Clinical significance and characterization of Streptococcus tigurinus isolates in an adult population. J Clin Microbiol. 2015: 53:3574-9.

15. Duval X, Millot S, Chirouze C, Selton-Suty C, Moby V, Tattevin P, et al. Oral streptococcal endocarditis, oral hygiene habits, and recent dental procedures: a case-control study. Clin Infect Dis. 2017;64:1678-85.

Ready to submit your research? Choose BMC and benefit from:

- fast, convenient online submission

- thorough peer review by experienced researchers in your field

- rapid publication on acceptance

- support for research data, including large and complex data types

- gold Open Access which fosters wider collaboration and increased citations

- maximum visibility for your research: over $100 \mathrm{M}$ website views per year

At BMC, research is always in progress.

Learn more biomedcentral.com/submissions 